\title{
Computers \& Coffee: Computergestützte Sprachprodukti- onstests zur syntaktischen Variation des „unbestimmten Artikels vor Massennomen“ im „Wienerischen“
}

\author{
Ludwig Maximilian BREUER
}

\begin{abstract}
s
Im vorliegenden Beitrag werden Teilergebnisse aus Sprachproduktionstests zur syntaktischen Variable „unbestimmter Artikel vor Massennomen“ eines laufenden Dissertationsprojektes zur syntaktischen Variation in Wien vorgestellt. Dabei wird kurz auf das „Wienerische" eingegangen, woraufhin die direkten Erhebungsmethoden vorgestellt werden, die mit Unterstützung des Phonogrammarchivs der Österreichischen Akademie der Wissenschaften durchgeführt worden sind. Der Beitrag konzentriert sich auf die Gestaltung der Aufgaben aus dem Sprachproduktionstest und zeigt anschließend auf der Grundlage verschiedener Variablenbetrachtungen, inwiefern Variation in den erhobenen Daten festgestellt werden kann. Besonders der Steuerungsfaktor der generischen bzw. Portionenlesart bedingt unterschiedliche Variantenfrequenzen, aber auch Alter und formaler Bildungsabschluss der Gewährspersonen erweisen sich als beeinflussende Faktoren für die Variantenwahl.
\end{abstract}

This paper presents partial results of language production tests for the syntactic variable "indefinite article with mass nouns". The results are part of an ongoing dissertation project devoted to the study of syntactic variation in Viennese German. Outlining Viennese German, the paper then depicts the direct survey methods applied, which were conducted with the support of the Phonogrammarchiv, the Institute for Audiovisual Research and Documentation of the Austrian Academy of Sciences. The paper focuses on the design of the language production tests and shows to what extent variation can be found in the collected data: the frequency of the variants is particularly influenced by the interpretation of mass nouns as generic or as a unit, and likewise by the age and educational level of the informants.

\section{Keywords}

Sprachproduktionstest, syntaktische Variation, Regionalsprache, Wienerisch Language production test, syntactic variation, regional language, Viennese German 


\section{Einleitung}

Kaffee ist nicht nur ein elementarer Bestandteil wissenschaftlicher Arbeit (zumindest jener des Autors); Kaffee ist überdies ein Wort, das zu einer besonderen Gruppe von Wörtern des Deutschen gehört: den Massennomen. ${ }^{1}$ Diese Gruppe von Wörtern vereint die Eigenschaft, keine abgrenzbare "Form" zu haben und somit nicht zählbar zu sein, wodurch sie (im Normalfall) keinen Plural aufweisen, wie z. B. auch Wasser (vs. *Wassers), Milch (vs. *Milche), Durst (vs. * Durste) etc. Dies hat auch grammatische Folgen: Im Standarddeutschen (ugs. „Hochdeutsch“) hat diese Eigenschaft zur Folge, dass kein Indefinitartikel (d. h. „unbestimmter Artikel“ also ein bzw. eine) direkt vor ihnen auftreten sollte - wobei dies in nicht-standardsprachlichen Sprechformen (im Folgenden: Varietäten/Sprechlagen, s. u.) nicht immer der Fall ist:

1. Die Laura mag am liebsten Kaffee. (NV54f)

2. Ja die Laura mag gerne einen Kaffee. (BR27m) ${ }^{2}$

Während das „Auslassen“ des Artikels (oder setzen eines Null-Artikels) vor dem Massennomen Kaffee in 1) einer prototypisch standardsprachlichen Verwendung entspricht, ist 2) einer prototypisch bairisch-dialektalen Verwendung zuzuordnen. In beiden Beispielen wird ganz prinzipiell, $d . h$. generisch, auf den Stoff Kaffee referiert $-d$. h., dass nicht auf ein zählbares Objekt referiert wird, womit der unbestimmte Artikel im (zumindest geschriebenen) Standarddeutschen getilgt werden sollte. Das betrifft bspw. auch Individuativa wie Kaninchen und Fisch, die in einer generischen Lesart verwendet werden können (Ich esse am liebsten Fisch) (vgl. Strobel 2016). Dahingegen können auch Massennomen in einer Portionenlesart verwendet werden, wie in:

\section{Paul hat einen Kaffee bestellt. (MG49f)}

Hierbei wird davon ausgegangen, dass auf genau eine Portion Kaffee referiert wird, weshalb ein unbestimmter Artikel im Standarddeutschen prototypisch verwendet wird. Somit kann eine syntaktische Variable ausgemacht werden („unbestimmter Artikel vor Massennomen“), die je nach

1 Der hier verwendete Begriff wird gegenüber anderen deutschsprachigen Begriffen bevorzugt, da er dem englischen Terminus (mass nouns) nahe steht (weitere Erläuterung s. u.).

2 Die Beispiele sind Antworten aus den hier diskutierten Sprachproduktionstests, also direkt dem hier erstellten Korpus entnommen. In Klammern werden anonymisierte Gewährspersonenkürzel sowie Alter und Geschlecht ( $m=$ male $/ f=$ female) angegeben. 
grammatischen und semantischen Steuerungsfaktoren unterschiedliche Varianten (entweder mit oder ohne unbestimmten Artikel) aufweist. Typologisch stellt dieses Phänomen eine Auffälligkeit bairischer Varietäten/ Sprechlagen dar, die aber über den bairischen Sprachraum hinausgeht (vgl. Strobel 2016). ${ }^{3}$ Diese spezielle Variable, die unten noch genauer besprochen wird, soll im vorliegenden Beitrag als Beispiel für die Erforschung vertikaler syntaktischer Variation in Wien dienen. Denn wie sich die Melange durch ihre Mischung definiert, so stellt auch das "Wienerische“ (in der hier verwendeten Definition, s. u.) eine Mischung aus verschiedenen Erscheinungsformen der deutschen (und anderen, aber hier vernachlässigten) Sprache( $\mathrm{n}$ ) dar. Damit bietet Wien Linguist/inn/en ein ganz besonderes „sprachliches Biotop" (vgl. Breuer \& Glauninger 2011: 2), das sich durch hohe Dynamik, ebenso hohe Komplexität sowie Heterogenität auszeichnet.

Der vorliegende Beitrag stellt das mithilfe des Phonogrammarchivs erhobene Korpus aus einem laufenden und umfangreichen Dissertationsprojekt zur syntaktischen Variation der modernen Regionalsprache (vgl. Schmidt \& Herrgen 2011: 66) in Wien vor. Dabei sollen insbesondere Erhebungsund auf innen beruhende Analyseverfahren besprochen werden, welche teilweise von traditionellen (dialektologischen und stadtsprachenforschungsbezogenen) Herangehensweisen abweichen. Die direkte Erhebung erfolgte mit der Unterstützung des Phonogrammarchivs und wird dort auch (langzeit-)archiviert. ${ }^{4}$ Damit wird auch ein Teileinblick in das dort - nach Abschluss des Dissertationsprojekts - zur Verfügung stehende Material geboten und wie dieses in einem aktuellen Projekt verarbeitet wird. Hierfür wird im Folgenden eine Übersicht über das Dissertationsprojekt und den Untersuchungsgegenstand gegeben, insbesondere auch eine Beschreibung des für das Projekt erstellten gesprochensprachlichen Korpus. Da nicht alle Teilbereiche des Korpus genau vorgestellt werden können, wird zur Verdeutlichung des Untersuchungsgegenstandes der syntaktischen Variation in Wien ausführlich auf die Methode der Sprachproduktionstests (im Folgenden: SPT) eingegangen. Daraus werden die Aufgabensets zur Erhebung der eingangs erwähnten syntaktischen Variable ${ }^{5}$ „unbestimmter Artikel vor

3 Natürlich kann diese typologische Besonderheit auch im Vergleich mit anderen Sprachen bzw. Varietäten betrachtet werden (s. z. B. Zifonun 2007), was aber nicht innerhalb des hier vorgestellten Projekts geleistet werden kann, das sich mit vielen syntaktischen Variablen beschäftigt und sich auf die Variation innerhalb des Wienerischen konzentriert. Da die Daten allerdings im Phonogrammarchiv vorliegen werden, können sie direkt für sprachkontrastive Untersuchungen herangezogen werden.

4 An dieser Stelle ein großes Dankeschön für die tatkräftige und freundliche Unterstützung an Christian Huber, Johannes Spitzbart und Nadja Wallaszkovits.

5 Zur Problematik des Begriffs „syntaktische Variable“ s. Kallenborn (2016: 23-29). 
Massennomen" als Beispiel herangezogen. Abschließend werden Teilergebnisse zu den Aufgaben präsentiert.

\section{Wienerisch und Variation}

Wie bereits angedeutet, wird dem „Wienerischen“ (s. u.) (nicht nur) von Seiten der linguistischen Forschung große Bedeutung zugeschrieben. Ganz pragmatisch liegt dies an der historischen, aber auch gegenwärtigen Gewichtung der Stadt (vgl. Breuer 2015: 194): Nicht nur ist sie eine der ältesten Städte des deutschsprachigen Raums, zudem ist sie die größte und prestigereichste $^{6}$ Stadt Österreichs. Dadurch wird sie zum sprachlichen Dreh- und Angelpunkt des standardisierten österreichischen Deutsch (vgl. Moosmüller 1991: 22). Darüber hinaus ist Wien ein besonders „schillernder sprachlicher Lebensraum" (Breuer \& Glauninger 2012: 2), welcher variationslinguistisch betrachtet besonders spannend ist (vgl. Breuer 2015: 194f.). Das "Wienerische“, wie es hier betrachtet werden soll, ist nicht (mehr) nur ein klar definierter „Basisdialekt“, der überall im Alltag von den Bewohner/ inne/n Wiens verwendet wird, sondern ein gesamtheitliches Spektrum aus mehreren sozio-situativ potenzierten Varietäten und/oder Sprechlagen. Damit ist gemeint, dass Sprecher/innen des "Wienerischen“ je nach (Gesprächs-)Situation (bedingt z. B. durch Faktoren wie Gesprächspartner/ in, institutionelle Einbettung des Gesprächs, Rolle im Gespräch, Thema etc.) unterschiedliche Sprechweisen verwenden, die auf den verschiedenen linguistischen Systemebenen (Lautung, Wortwahl und -bildung sowie Satzbau) unterschiedlich stark voneinander abweichen. Dieser Einsatz von Varietäten ist dabei äußerst dynamisch und somit ständigem Wandel unterzogen.

Unter „Wienerisch“ soll somit im Folgenden eine moderne Regionalsprache (im Sinne von Schmidt \& Herrgen 2011) verstanden werden, also ein komplexes sprachliches System bestehend aus mehreren Teilsystemen (Varietäten mit je mehreren Sprechlagen), das von Wiener/inne/n als „in Wien üblich" verstanden wird (vgl. Breuer 2015: 195). Hierbei werden „Varietäten“ verstanden „als partiell systemisch differente Ausschnitte des komplexen Gesamtsystems Einzelsprache" (Schmidt \& Herrgen 2011: 51). Sprechlagen dagegen werden aufgefasst als „Verdichtungsbereiche variativer Sprachverwendung " (ibid.: 52). Dabei wird davon ausgegangen, dass die Sprechlagen (als Teil einer oder mehrere Varietäten - der Status ist im Wienerischen nicht geklärt, wie in Breuer 2012 beschrieben) zwischen den Polen

6 Wobei „prestigereich“ hierbei natürlich nicht heißt, dass die Stadt, ihre Bewohner/innen und ihre sprachlichen Varietäten überall in Österreich immer nur „positive“ Assoziationen hervorrufen. Das ökonomische, politische sowie mediale (Presse- und Medienagenturen) und somit soziolinguistisch relevante Prestige ist aber kaum abzustreiten. 
„intendierter Dialekt“ und „intendierter Standard“ variieren, d. h. zwischen bestimmten Konzepten des Dialekts bzw. Standards, die von Sprechenden mehr oder weniger angestrebt, aber sprachlich (real) nie ganz erreicht werden (vgl. Glauninger 2012: 111). Der intendierte Dialekt wird dem ostmittelbairischen Dialektraum zugeordnet, womit die unten besprochenen Varianten der syntaktischen Variablen stets dem (gesprochenen) österreichischen Standarddeutsch ${ }^{7}$ bzw. bairischen Dialekten zuzuordnen sind.

Wie erläutert stellt das Wienerische somit einen bedeutsamen und sehr heterogenen Untersuchungsgegenstand dar. Dabei verwundert es vielleicht, dass eine "umfassende Stadtsprachenuntersuchung, die alle nebeneinander existierenden Varietäten und Fremdsprachen berücksichtigt" (Tatzreiter 2002: 128), ein Forschungsdesiderat bleibt. Zudem wurden bisher vor allem phonetisch-phonologische (z. B. Moosmüller 1987), teilweise lexikalisch(-pragmatische) (z. B. Glauninger 2012) und nur selten syntaktische (z. B. Glauninger 2008, 2011 und insbesondere Patocka 1997) Fragestellungen aufgegriffen. Prinzipiell ist in variationslinguistischen Untersuchungen, die nicht nur auf die horizontale (areale), sondern insbesondere auf die vertikale (situative) Variation abzielen, die Syntax ein aufzuholendes Forschungsdesiderat (vgl. Kallenborn 2016: 20-23). Ebenso unklar wie die syntaktischen Merkmale des Wienerischen ist dessen Varietätenspektrum (vgl. Breuer 2015: 191-194). Das bereits erwähnte Dissertationsprojekt versucht, diese Forschungsdesiderate anzugehen, indem es auf Grundlage der syntaktischen Variation zwischen den Polen „,intendierter Dialekt“ und „intendierter Standard“ die Variationsstruktur des Wienerischen situationsabhängig auf empirischer Basis erhebt, beschreibt und analysiert.

\section{Korpus und Methoden}

Im Folgenden wird kurz auf das Projektkorpus sowie die im Projekt allgemein angewendeten Methoden eingegangen. Insgesamt setzt das Dissertationsprojekt auf ein „multidimensionales Methodenset“ (Kallenborn 2016: 41-43). Im vorgestellten Projekt setzt sich das Gesamtdatenkorpus zusammen aus:

7 Nicht hinreichend linguistisch geklärt ist dabei, was das österreichische Standarddeutsch auf syntaktischer Ebene ausmacht bzw. von anderen deutschsprachigen Standardvarietäten unterscheidet. Untersuchungen dazu stellen derzeit v. a. das Projekt „Variantengrammatik des Standarddeutschen“ an (vgl. <http://www.variantengrammatik.net/index.html>). Auch das Konzept des intendierten Standards der Sprechenden ist nicht immer klar, wobei Moosmüller (1991) einen tiefgehenden Einblick in das Verhältnis von österreichischem Standard und österreichischen Dialekten liefert. Peter (vgl. 2015: 137-144) kann zeigen, dass sich österreichische (sowie schweizerische) Sprecher/innen im Vergleich zu bundesdeutschen nicht nur durch eine erhöhte Form- und Normbewusstheit auszeichnen, sondern dem bundesdeutschen Standard auch ein hohes Prestige zuschreiben. 
- bestehenden Korpora (siehe z. B. Breuer i. V.),

- einem selbsterstellten spontansprachlichen Korpus (Interviews und Freundesgespräche),

- Sprachproduktionstests (Standard- und Dialektdurchlauf) und

- (Online-)Fragebögen (siehe z. B. Breuer 2015, 2016).

Der Fokus im vorliegenden Beitrag liegt auf den SPT (genaue Beschreibung s. u.). Die SPT wurden im Rahmen der spontansprachlichen Erhebungen durchgeführt und stellen mit ihnen gemeinsam das direkt erhobene ${ }^{8}$ Korpus dar. Im Gegensatz zu den spontansprachlichen Daten, in denen die Gewährspersonen von mir interviewt worden sind (Fragen zur Person, zur Spracheinstellung etc.) oder zwei Gewährspersonen ohne meine Anwesenheit vorgegebene Themen (Verhältnis zum Wienerischen, aber auch "Kochen" etc.) besprochen haben, handelt es sich bei den SPT um elizitierte Daten, die mithilfe eines standardisierten Verfahrens erhoben worden sind. Prinzipiell werden den Gewährspersonen bei den SPT mithilfe eines Computers verschiedene (visuelle oder auditive) Stimuli präsentiert und dazu ebenfalls vom Computer abgespielte Aufgaben gestellt. Ein Beispiel hierfür wird im vorliegenden Beitrag anhand der Aufgaben zum „unbestimmten Artikel vor Massennomen" geliefert, wie unten genau erläutert wird.

Durch die Unterstützung des Phonogrammarchivs der Österreichischen Akademie der Wissenschaften bei der Erstellung dieses Korpus wurden die Aufnahmen in einer hohen (und mit anderen Aufnahmen des Phonogrammarchivs vergleichbaren) Qualität aufgezeichnet und werden dort (nach Abschluss des Projekts) für andere wissenschaftliche Fragestellungen zur Verfügung stehen. Alle anderen (auch Analyse-)Daten werden nach Abschluss der Dissertation zumindest in Form einer Datenbank (wenn möglich auch online) zur Verfügung gestellt.

Insgesamt wurden Sprachaufnahmen von 32 Gewährspersonen (im Folgenden: GWP) aufgezeichnet, welche alle in Wien geboren sowie aufgewachsen sind und von denen mindestens ein Elternteil ebenfalls in Wien geboren und aufgewachsen ist. Das GWP-Setting wird in drei Altersgruppen gegliedert, und diese in Subgruppen nach formalem Bildungsabschluss (+/- Hochschulreife):

8 Mit „direkt erhobenen Daten“ ist gemeint, dass ein Explorator vor Ort ist und Aufzeichnungen entweder schriftlich oder mithilfe eines Tonbandgerätes durchführt (vgl. Niebaum \& Macha 2006: 15). Auch die Freundesgespräche werden, aufgrund des auditiven Aufnahmegerätes, das vom Explorator platziert wurde, als „direkt“ bezeichnet (kurze terminologische Diskussion s. Kallenborn 2016: 64). 


\begin{tabular}{|c|c|c|}
\hline \multirow{2}{*}{ Altersgruppe } & Hochschulreife & Anzahl GWP \\
\hline \multirow{2}{*}{$21-35$} & - & 4 \\
\cline { 2 - 3 } & + & 8 \\
\hline \multirow{2}{*}{$36-60$} & - & 4 \\
\cline { 2 - 3 } & + & 4 \\
\hline \multirow{2}{*}{$>60$} & - & 4 \\
\hline
\end{tabular}

Tab. 1: GWP-Gruppen

Aus der Zusammenstellung des GWP-Settings wird ersichtlich, dass ein Schwerpunkt auf die jüngeren GWP mit Hochschulreife und die mittlere Altersgruppe ohne Hochschulreife gelegt wird. Das liegt daran, dass der Vergleich dieser beiden Gruppen eine besonders hohe Dynamik verspricht; zudem können insbesondere für Daten zur ältesten Gruppe ggf. bereits bestehende Korpora herangezogen werden. Prinzipiell wird das Setting einerseits traditionell-dialektologischen Ansprüchen gerecht (es werden sog. NORMs bzw. NORFs befragt), gleichzeitig wird der Untersuchung der vertikalen Variation dadurch Genüge getan, dass auch jüngere Gewährspersonen befragt werden. Insgesamt wurden 12 Frauen und 20 Männer befragt - eine gleichmäßige Aufteilung auf die biologischen Geschlechter war aus forschungspragmatischen Gründen (GWP-Akquise) leider nicht möglich. Dieses Ungleichgewicht in den Daten wird quantitativ aber durch den Online-Fragebogen ausgeglichen, der vorwiegend von Frauen beantwortet wurde. Bewusst wurde darauf verzichtet, die GWP über alle 23 Wiener Gemeindebezirke verteilt auszuwählen, da dies ebenfalls forschungspragmatische Probleme hervorgerufen hätte. Darüber hinaus ist die horizontale Variation innerhalb Wiens linguistisch kaum mehr anzunehmen (vgl. Moosmüller 1987: 30 sowie Tatzreiter 2002: 136). Die GWP wohnten zum Zeitpunkt der Erhebungen in 15 unterschiedlichen Gemeindebezirken, was als gute Streuung betrachtet werden kann. ${ }^{9}$ Die Interviews fanden bis auf zwei Ausnahmen am Wohnort der jeweiligen GWP statt, die Freundesgespräche bis auf eine Ausnahme am Wohnort einer der beiden teilnehmenden GWP. Je Person wurden zwei Erhebungstermine vereinbart:

9 Konkret in Wien 1030, 1050, 1070, 1090, 1100, 1110, 1120, 1130, 1140, 1150, 1160, 1180, 1200, 1210 und 1220. 
1. Interview (I) + Standarddurchlauf Sprachproduktionstest (SPT-S)

2. Gelenktes Freundesgespräch (GFG) + Dialektdurchlauf Sprachproduktionstest (SPT-D)

Je Erhebung wurden zunächst die spontansprachlichen Daten erhoben, woraufhin die SPT stattfanden. ${ }^{10}$ Das Interview zielte dabei - im Gespräch mit dem fremdem, (süd-)deutschen Standard sprechenden Explorator ${ }^{11}$ - auf möglichst standardnahe Varietäten/Sprechlagen ab, während das gelenkte Freundesgespräch - Gespräch mit einer zweiten Gewährsperson ohne Anwesenheit des Explorators - dialektnahe Varietäten/Sprechlagen evozieren sollte. ${ }^{12} \mathrm{Um}$ beim ersten Termin ein möglichst formelles Setting zu evozieren, wurde von Seiten des Explorators auf Siezen bestanden, während zur Initiierung einer informellen Situation geduzt wurde. Insgesamt wurden in den Erhebungen rund 104 Stunden Audiomaterial aufgezeichnet, das sich wie in Tab. 2 dargestellt zusammensetzt.

\begin{tabular}{|c|c|c|}
\hline Methode & Anzahl & Dauer in hh:mm \\
\hline I & 32 & $43: 33$ \\
\hline GFG & 15 & $16: 10$ \\
\hline SPT-S & 32 & $23: 20$ \\
\hline SPT-D & 32 & $21: 21$ \\
\hline
\end{tabular}

Tab. 2: Übersicht Audiomaterial aus direkten Erhebungsmethoden

Insgesamt wurden in den SPT-Durchläufen je 70 Aufgaben und eine Nacherzählung bestehend aus 8 Text- (nur auditive Stimuli), 40 Bild- (Bildstimuli, z. B. s. u. EM1 + EM2) und 22 Videoaufgaben (z. B. s. u. EM3) erhoben, wobei 11 Primär-Variablen, d. h. die Variablen, für die die Aufgaben gezielt erstellt worden sind, abgefragt wurden.

Im Phonogrammarchiv werden neben den Aufnahmen auch die Protokolle, die Transkripte (zu den spontansprachlichen Daten) sowie weitere

10 Nur für zwei GWP konnte es nicht eingerichtet werden, dass sie miteinander oder mit einem $/ \mathrm{r}$ zweiten Wiener/in ein Freundesgespräch durchführen. Bei diesen Personen wurde beim zweiten Termin zunächst ein kurzes, unbefangenes Gespräch zur Evokation eines informellen Settings geführt, woraufhin der SPT durchgeführt wurde.

11 Die Erhebungen wurden ausschließlich von mir durchgeführt.

$12 \mathrm{Zu}$ Verwendung von Interviews und Freundesgesprächen als Methode der Erhebung verschiedener Variationspole s. Lenz (2003: 57). 
Unterlagen zur Erhebung nach Abschluss des Projekts zur Verfügungen stehen.

\section{Artikel und Massennomen}

Eine der oben genannten Primär-Variablen ist der „unbestimmte Artikel vor Massennomen", die im vorliegenden Artikel als Beispiel für die Fragestellung, Methoden und Analysen der Dissertation herangezogen wird. Konkret geht es dabei darum, in unterschiedlichen Lesarten (generische Lesart s. 1) und 2) vs. Portionenlesart s. 3)) die Realisierung von Massennomen zu evozieren und zu untersuchen, in welcher Situation und in welcher Lesart ein unbestimmter Artikel vor Massennomen realisiert wird. Die Aufgaben dazu werden im nächsten Kapitel genau geschildert. Hier werden die - zumindest für die gebotene Auswertung relevanten - Hintergründe für die syntaktische Variable rudimentär beschrieben. Ohne dabei auf unterschiedliche Theorien zu Funktion und Stil des Artikelgebrauchs einzugehen, ${ }^{13}$ sei zumindest erwähnt, dass die Bezeichnungen "bestimmter"/,definiter" (der, die, das) bzw. „unbestimmter"/,indefiniter" Artikel (ein, eine bzw. Null-Artikel für den Plural) problematisch sind, da sie nur auf die referenzsemantische Funktion der Determination von Bestimmbarkeit (oder auch: Bekanntheit) referieren (Hast du (einen) Kaffee? vs. Hast du den Kaffee?). Darüber hinaus hat die Artikelverwendung aber auch morphologisch-syntaktische (z. B. Markierung des Genus in Der Geschmack eines/des Kaffees vs. Der Geschmack Kaffees), kommunikativ-pragmatische (z. B. Markierung besonderer Intensität, wie in: Das ist aber ein (guter) Kaffee) sowie stilistische Funktionen (vgl. z. B. Kolde 1989: 27 - so wie bspw. die Verwendung je nach Situation standardnah vs. standardfern). Das heißt: Es kann nicht per se davon ausgegangen werden, dass der Artikelgebrauch ausschließlich davon abhängt, ob das Referenzobjekt „,bestimmt" oder „unbestimmt“ bzw. „bekannt" oder „unbekannt“ ist. Für die Gestaltung von Sprachproduktionstests, die auf die Verwendung von Artikeln abzielen, bzw. für die Analyse des Artikelgebrauchs sind also ggf. auch diese Faktoren zu berücksichtigen. In der vorliegenden Untersuchung sind vor allem der Indefinitartikel (im Folgenden: EIN-) und der Null-Artikel (im Folgenden: 0-) von Bedeutung. ${ }^{14}$ Wichtig für den Artikelgebrauch beim Vergleich von standardnahen und bairischen Varietäten/Sprechlagen ist, dass vom Bairischen behauptet wird,

13 Genauere Ausführungen dazu s. Breuer (2015: 197f.).

14 Auch definite Artikel treten in den Ergebnissen zu den SPT auf, insbesondere im Videoexperiment (s. u.), in denen der Textaufbau der Antwort dies bedingt. Diese werden selbstverständlich annotiert und in der Analyse betrachtet, sind aber für die hier beschriebenen Ergebnisse vernachlässigbar. 
dass es einen sehr konsequenten und durchgehenden Artikelgebrauch aufweist (d. h. häufig Artikel setzt bzw. selten 0- setzt; vgl. Weiß 1998: 69f.), wohingegen das Standarddeutsche in einer höheren Frequenz 0- setzt, z. B. vor Eigennamen oder eben vor Massennomen.

Unter den bereits eingangs erwähnten Massennomen (auch Kontinuativa oder Nicht-Individuativa) werden üblicherweise transnumerale Nomen verstanden, also Nomen, die keine räumliche Diskretheit aufweisen und damit nicht zählbar sind (vgl. Gallmann 1990: 28-31). Darunter fallen zumindest prototypisch zwei unterschiedliche Gruppen von Nomen: Stoffbezeichnungen (Milch, Geld, Wasser) und Abstrakta (Hunger, Durst, Lärm). ${ }^{15}$ Durch ihre Eigenschaft, dass sie nicht zählbar sind, ergeben sich (zumindest in standarddeutschen Varietäten) verschiedene morphosyntaktische Besonderheiten der Massennomen, insbesondere weisen sie keine Pluralmorphologie auf ( ${ }^{*}$ Durste); Stoffbezeichnungen können nur über die Verwendung von z. B. Maßangaben mit Numeralia kombiniert werden (drei Gläser Milch vs. *drei Milche) und treten obligatorisch ohne Artikel bzw. mit 0- auf (Ich habe (*einen) Durst) ${ }^{16}$ (s. z. B. Krifka 1991, Chierchia 1998, Strobel 2016). Vor allem bei Stoffbezeichnungen ist aber zu bedenken, dass fast alle in einer Sorten- bzw. Artenlesart zumindest im fachsprachlichen Gebrauch eine Pluralform aufweisen (verschiedene Weinsorten/Weine), wobei verschiedene Pluralformen unterschiedlich stark konventionalisiert sind. ${ }^{17}$ Wie bereits erwähnt, kommt hinzu, dass Massennomen in einer Portionenlesart auch als Individuativa interpretiert werden und somit mit EINauftreten können (Er bestellt ein Wasser), wobei direkt auf eine bestimmte Quantität des Stoffes/Materials referiert wird. Prinzipiell kann festgehalten werden, dass eine Unterscheidung von Massennomen und Individuativa häufig strittig sein kann; so können auch Individuativa zur Bezeichnung des Materials herangezogen werden (Lamm schmeckt gut), was zur Hypothese führen kann, „dass eigentlich alle Substantive bis zu einem gewissen Grad \pm COUNT bzw. \pm MASS sein können“ (Strobel 2016). Im vorliegenden Beitrag

15 Außerdem werden häufig diverse Kollektiva wie Obst, Geflügel, Publikum hinzugezählt, die eine Gruppe von mehreren einheitlichen (diskreten) Dingen repräsentieren und hier vernachlässigt werden.

Die Markierung besonderer Intensität bildet hier z. B. eine Ausnahme, wenn also jemand besonders großen Durst hat, kann EIN- durchaus auftreten (vgl. Kolde 1989: 137f.).

17 Eine Korpus-Untersuchung zur Frequenz von Pluralen diverser Stoffbezeichnungen wäre wünschenswert. So ist Kaffees (im nicht-fachsprachlichen Gebrauch) sicherlich häufiger in Verwendung als Milche, wobei auch Letzteres durchaus auftritt, z. B.: „Aber nicht nur die Milche auch die anderen Produkte der Gmundner Molkerei werden im Ausland zunehmend geschätzt." (Oberösterreichische Nachrichten, 20.3.2001, s.: <https://www.wiso-net.de/document/OOEN_srv20010320681854>; 22.7.2016).) 
werden Stoffbezeichnungen prinzipiell als Massennomen betrachtet, die in verschiedenen Lesarten unterschiedliche grammatische Eigenschaften aufweisen. Die Lesart wird durch den Kontext (der Einzelaufgaben s. u.) und das Prädikat (bestellen vs. mögen / am liebsten trinken) bestimmt, da Letzteres vorgibt, ob von einer generischen oder einer Portionenlesart ausgegangen werden kann (vgl. Grammis 2005).

Wie bereits erwähnt, wird der Artikelgebrauch von 0- und EIN- in den standardnahen bzw. bairischen Zielvarietäten/-sprechlagen in den verschiedenen Lesarten fokussiert. Während im Standarddeutschen 0- (zumindest in der generischen Lesart) vor Massennomen ausgeschlossen wird (wie in 1) und 2)), kann in bairischen Varietäten EIN- gesetzt werden (wie in 3)) (s. Glaser 1996, Breuer 2015, Strobel 2016). Das heißt, dass die syntaktische Variable EIN- vor Massennomen unter Berücksichtigung verschiedener Steuerungsfaktoren (Lesart, bestimmtes Nomen, Attribuierung des Nomens) untersucht wird. Dabei wird davon ausgegangen, dass in generischen Kontexten in standardnahen Varietäten/Sprechlagen (evoziert durch bestimmte Situationstypen s. u.) 0- vor Massennomen bevorzugt verwendet wird, in standardfernen Varietäten/Sprachlagen hingegen die Variante mit EIN-. Außerdem wird überprüft, ob die EIN-Variante in generischen Kontexten vermieden wird und inwiefern sich die Wahl des Nomens auf die Variantenwahl auswirkt.

\section{Sprachproduktionstests}

Die Datenquellen für die hier präsentierten Ergebnisse stammen aus sog. Sprachproduktionstests, ${ }^{18}$ die speziell zur Erhebung syntaktischer Variation in der Vertikalen in Wien entwickelt worden sind. Prinzipiell sind die Sprachproduktionstests so aufgebaut, dass mittels eines mobilen Computers (in diesem Fall ein aufgestelltes Tablet) verschiedene Aufgaben präsentiert werden. Dabei werden medial unterschiedliche Stimuli (schriftliche, auditive, visuelle) am Gerät wiedergegeben, zu denen wiederum vom Gerät auditiv Aufgaben gestellt werden. Es wird z. B. ein Video gezeigt, in dem ein Mann Geld abhebt, woraufhin die Aufgabe „Beschreiben Sie möglichst genau, was der Mann im folgenden Video tut." zu hören ist. Die genauen Aufgabenstellungen (je Aufgabenset) sowie die entsprechenden visuellen Stimuli werden unten beschrieben. Die GWP sollten dann mündlich darauf antworten. Da auf die Variationspole „intendierter Standard“ und

18 Kallenborn (vgl. 2016: 70) plädiert zu Recht für eine Bevorzugung des Begriffs „Sprachproduktionstest“ im Gegensatz zu „Sprachproduktionsexperiment“, der den Umstand berücksichtigt, dass der Methode (in der hier gegebenen Anwendung) zentrale Elemente strenger Experimentsettings fehlen. 
„intendierter Dialekt" abgezielt wird, sind die Aufgabenstellungen und Stimuli im Standarddurchlauf (SPT-S, s. o.) in einer standardnahen Varietät/Sprechlage, im Dialektdurchlauf (SPT-D) in einer dialektalen Varietät/ Sprachlage des Wienerischen gehalten. ${ }^{19}$ Diese Herangehensweise für Fragestellungen der vertikalen Variation wurde von Kallenborn (s. 2011, 2016) für die Sprache im moselfränkischen Ort Graach an der Mosel ${ }^{20}$ entwickelt und erstmalig an einem breiten Spektrum syntaktischer Variablen durchgeführt. Im Gegensatz zu den Erhebungen von Kallenborn für den ruralen Raum wurden die GWP in der vorliegenden Untersuchung nicht darum gebeten, „Hochdeutsch“ respektive „Platt“ (in Graach übliche Bezeichnung für den Dialekt) zu sprechen (vgl. Kallenborn 2016: 67), sondern nur darum gebeten, so zu sprechen, wie sie mit einem „Fremden“ bzw. einem "guten Freund" reden würden. Dies trägt der äußerst dynamischen sprachlichen Situation im urbanen Raum (s. o.) Rechnung (vgl. Dingeldein 2002: 22, Breuer 2012: 8f.), in dem (auch insbesondere in Wien, s. o.) nicht von klaren Konzepten zu den Begriffen „Hochdeutsch" und „Dialekt" bzw. „Wienerisch“ ausgegangen werden kann. ${ }^{21}$ Die Aufgaben wurden zur Vermeidung von Wiederholungseffekten über die einzelnen GWP hinweg in einer randomisierten Reihenfolge gestellt, wobei der Explorator lediglich das Starten bzw. Wiederholen einer Aufgabe via Funktastatur vornahm. Bei Unklarheiten wurden vom Explorator Rückfragen gestellt bzw. beantwortet. ${ }^{22}$

Im Folgenden wird kurz allgemein auf die Vor- und Nachteile dieser Methode eingegangen. Ausführlich werden sie in Kallenborn (2016: 64-70) beschrieben, woran sich die Darstellung hier orientiert. Der wichtigste Vorteil der SPT ist sicherlich, dass gerade in dialektologischen Forschungen betrachtete syntaktische Variablen in freien Gesprächen relativ selten auftreten (s. z. B. Kortmann 2010: 844f., Patocka 1989: 51), wodurch eine

19 Für die Aufnahmen wurden zwei Sprecher aus Wien herangezogen, die denselben Kriterien wie die GWP entsprechen, aber nicht an der Erhebung teilgenommen haben.

20 Zur genauen Evokation unterschiedlicher Varietäten/Sprechlagen in den SPT durch die Erhebungssituation s. Kallenborn 2016: 66-68; im Vergleich dazu wurde dieses Konzept in der vorliegenden Untersuchung durch eine strikte Trennung der Aufnahmesituationen (s. o.) und die Einbindung sprachlicher Distanz- (Siezen) bzw. Näheformen (Duzen) sowie die Verwendung schriftlicher Stimuli abgewandelt.

21 In den Interviews werden die GWP zu ihren Konzepten befragt, wobei eben jene Komplexität der unterschiedlichen Vorstellung festgestellt werden kann, die zuvor schon in einem Online-Fragebogen abgefragt wurde (s. Breuer 2015, 2016). Eine genaue Auswertung dieser Spracheinstellungsfragestellungen liegt noch nicht vor, wird aber in der Dissertation angestrebt.

22 In der Dateneingabe werden dabei „spontane“, d. h. direkt nach der Aufgabenstellung am Computer gegebene Antworten, von „beeinflussten“, d. h. Antworten, die erst nach Rückfrage oder durch Einflussnahme durch den Explorator gegeben worden sind, unterschieden. 
große Datenmenge freier Gespräche nötig wäre, um überhaupt statistische Aussagen über das Auftreten verschiedener Varianten zu tätigen. Überdies ermöglichen standardisierte Verfahren, die an allen GWP durchgeführt werden, die Vergleichbarkeit der Daten, und zwar intraindividuell, interindividuell und auch über verschiedene Erhebungen bzw. Untersuchungen hinweg. Prinzipiell gilt dies für alle standardisierten Testverfahren (s. bspw. Reinecke 2014), wie z. B. auch für Fragebögen: ${ }^{23}$ Durch die Evokation bestimmter Phänomene in einem gleichbleibenden Setting und mit denselben Aufgaben(kontexten) werden kontrastive Aussagen über die Wahl differenter Varianten (von verschiedenen GWP oder ein und derselben GWP) mit ausreichender Quantität ermöglicht. Was die syntaktischen Phänomene selbst betrifft, können in standardisierten Verfahren, in denen die (situativen und sprachlichen) Kontexte manipuliert werden können, verschiedene (z. B. von der Forschungsliteratur postulierte) sprachliche Steuerungsfaktoren der Variantenwahl überprüft werden (s. z. B. Lenz 2008: 163). Im Gegensatz zu Fragebögen werden die (Fragen-)Stimuli - wie auch die Antworten - beim SPT auditiv wiedergegeben. ${ }^{24}$ Dies ist insofern von Vorteil, da die Abstraktionsebene der Schrift zur Erhebung gesprochensprachlicher Phänomene entfällt (s. Fleischer, Kasper \& Lenz 2012: 30-32). Natürlich ergeben sich auch Nachteile aus dieser Methode - der wichtigste ist sicherlich die Artifizialität der Erhebungssituation: Da die meisten Menschen (zumindest nicht regelmäßig und nicht „natürlich“) mit Computern sprechen, ist dies keine Alltagssituation, evoziert also keine „natürliche“ Gesprächssituation. ${ }^{25}$ Insbesondere wenn es, wie in diesem Vorhaben, um die situative Variation geht, ist diesem Umstand natürlich Rechnung zu tragen und die Methode entsprechend anzupassen bzw. dies in der Analyse zu berücksichtigen. In jedem Fall überwiegen die Vorteile dieser Methode bei Weitem; außerdem lassen sich die Nachteile durch ein multidimensionales Methodenset (s. o.) ausgleichen.

\section{Aufgabensets zur Variable „unbestimmter Artikel vor Massennomen“}

Insgesamt wurden zur Variable „unbestimmter Artikel vor Massennomen" drei Aufgabensets entwickelt, welche alle auf Stoffbezeichnungen

23 Standardisierte Verfahren für syntaktische Fragestellungen erweisen sich in verschiedenen Projekten als valide und äußerst ergiebig (s. etwa Lenz 2008; Kallenborn 2011; Fleischer, Kasper \& Lenz 2012).

24 Zumindest trifft dies auf „Offline-Fragebögen“ zu; bei Online-Fragebögen ist dies durchaus fruchtbringend möglich (vgl. Breuer 2015: 211). 
abzielen, da Abstrakta relativ schwierig darzustellen erschienen. Die Aufgabensets bestehen aus mehreren Aufgaben, die sich durch ihren konkreten visuellen Stimulus, der die Primär-Variable betrifft, unterscheiden, wobei eine Aufgabe einmal im SPT-S und zudem im SPT-D gestellt wird. Das heißt, dass sich der visuelle Stimulus vom SPT-S im SPT-D wiederholt - um dabei aber Wiederholungseffekte zu vermeiden, wurde er in Aspekten, die die Variantenwahl nicht beeinflussen sollten, verändert. So wurden bspw. die Protagonist/inn/en der Videos getauscht, wobei die gleiche Handlung mit demselben Stoff durchgeführt wird. ${ }^{26}$ Tabelle 3 zeigt eine Übersicht der Aufgabensets:

\begin{tabular}{|c|c|c|c|}
\hline Aufgabenset & $\begin{array}{c}\text { Aufgaben je } \\
\text { Durchlauf }\end{array}$ & Visueller Stimulus & Steuerungsfaktor \\
\hline EM1 & 3 & Bild & +generisch \\
\hline EM2 & 3 & Bild & +portion \\
\hline EM3 & 4 & Video & +/-portion \\
\hline
\end{tabular}

Tab. 3: Übersicht Aufgabensets

Die ersten beiden Aufgabensets sind komplementär zu verstehen, da sie in einem sehr ähnlichen Kontext (s. u.) eine generische (Handlung: „mögen“) bzw. eine Portionenlesart (Handlung: „bestellen“) evozieren. Das Aufgabenset EM3 dagegen evoziert, abhängig von der Handlung, unterschiedliche Lesarten; außerdem soll es durch die Beschreibung eines Videos längere, freiere Antworten evozieren und somit die Massennomen in einem anderen Kontext abfragen. Je Aufgabe werden zudem unterschiedliche Stoffbezeichnungen evoziert, sodass auch die Verwendung der unbestimmten Artikel in Bezug auf die Semantik der Stoffbezeichnungen betrachtet werden kann - eine Übersicht über die im Folgenden analysierten Aufgaben und die entsprechenden visuellen Stimuli-Inhalte (Stoffbezeichnung, handelnde Personen, Handlung) findet sich in Tabelle 4. 


\begin{tabular}{|c|c|c|c|c|}
\hline Aufgabenset & Aufgabe & Person & $\begin{array}{l}\text { Stoffbezeich- } \\
\text { nung }\end{array}$ & Handlung \\
\hline \multirow{6}{*}{ EM1 } & SPT-S1 & Jakob & \multirow{2}{*}{ Milch } & \multirow{6}{*}{ mögen } \\
\hline & SPT-D1 & Paul & & \\
\hline & SPT-S2 & Sabine & \multirow{2}{*}{ Kaffee } & \\
\hline & SPT-D2 & Laura & & \\
\hline & SPT-S3 & Julia & \multirow{2}{*}{ Wasser } & \\
\hline & SPT-D3 & Alex & & \\
\hline \multirow{6}{*}{ EM2 } & SPT-S1 & Julia & \multirow{2}{*}{ Milch } & \multirow{6}{*}{ bestellen } \\
\hline & SPT-D1 & Laura & & \\
\hline & SPT-S2 & Alex & \multirow{2}{*}{ Kaffee } & \\
\hline & SPT-D2 & Paul & & \\
\hline & SPT-S3 & Sabine & \multirow{2}{*}{ Wasser } & \\
\hline & SPT-D3 & Jakob & & \\
\hline \multirow{4}{*}{ EM3 } & SPT-S1 & Eine Frau & \multirow{2}{*}{ Geld } & \multirow{2}{*}{ abheben } \\
\hline & SPT-D1 & Ein Mann & & \\
\hline & SPT-S2 & Eine Frau & \multirow{2}{*}{ Leder/Stoff } & \multirow{2}{*}{ schneiden } \\
\hline & SPT-D2 & Ein Mann & & \\
\hline
\end{tabular}

\section{Tab. 4: Überblick ausgewertete Aufgaben}

Die Aufgabensets EM1 und EM2 sind, wie bereits erwähnt, parallel aufgebaut. Bei beiden Aufgaben werden zwei Bilder nebeneinander gezeigt: Links ist eine Person mit einem Namen abgebildet, ${ }^{27}$ rechts ein Stoff (siehe Abb. 1).

27 Die Angabe eines Namens evoziert, dass eben jener Name bei der Antwort verwendet wird; dadurch kann sekundär der Einsatz des bestimmten Artikels vor Eigennamen (wie in vielen anderen Aufgabensets) untersucht werden. 


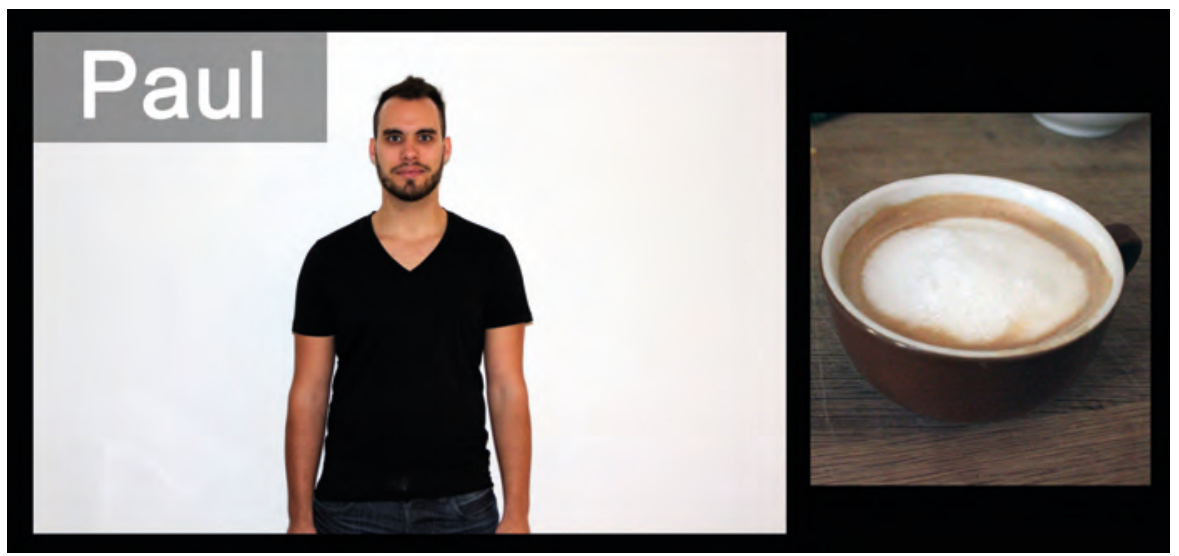

Abb. 1: Beispiel für Bildaufgabe EM2 SPT-D2

Dabei wurde bei EM1 die Herausforderung angenommen, einen Stoff unabhängig von möglichen Behältern auf einem Foto darzustellen, um sich möglichst weit von der Portionenlesart zu entfernen. Dafür werden Kaffeebohnen (ohne erkennbare Anzahl) bzw. Wasser/Milch abgebildet, das/die gerade von einem nicht komplett abgebildeten Behälter in einen anderen geschüttet wird (s. Abb. 2).
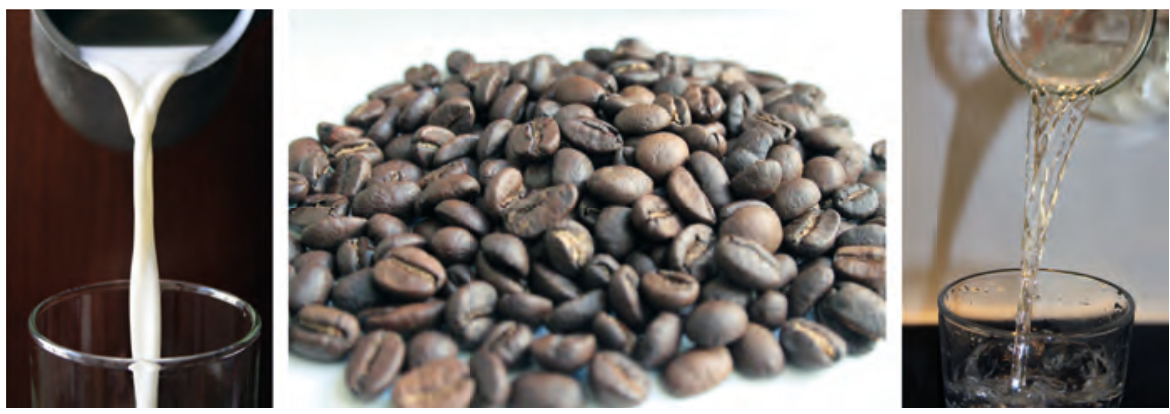

Abb. 2: Abbildungen generisch (Milch, Kaffee, Wasser)

In EM2 werden sie im Glas bzw. in einer Tasse (s. Abb. 1) abgebildet. Als Aufgabenstellung, d. h. als konkrete auditive „Fragestellung“, wird jeweils auditiv ein Kontext sowie die Aufgabenstellung (in der entsprechenden Varietät, s. o.) vorgegeben; das Bild unterscheidet sich dabei je Aufgabe wie oben in der Tabelle angegeben: 
- EM1: Auf den folgenden Bildern sehen Sie eine Person mit Ihrem Lieblingsgetränk. [Bild wird eingeblendet] Erzählen Sie einem Freund, was die Person am liebsten mag. Bilden Sie dabei einen ganzen Satz.

- EM2: Auf den folgenden Bildern sehen Sie eine Person, die gerade etwas bestellt. Daneben, was Sie bestellen will. [Bild wird eingeblendet] Erzählen Sie einem Freund, was die Person bestellt hat. Bilden Sie dabei einen ganzen Satz.

Durch diese Vorgaben sollen eindeutig generische Kontexte (Lieblingsgetränk ist unabhängig von der Portion) bzw. Portionenlesarten evoziert werden (es werden immer Portionen bestellt). Wie die Ergebnisse zeigen werden (s. u.), wurde der gewünschte Effekt erzielt.

Bei EM3 dagegen werden eine Aufgabenstellung und dann ein Video präsentiert, je Aufgabe unterscheidet sich hier ausschließlich das Video. Das Video zeigt Personen (ohne Angabe von Namen), die die oben genannten Handlungen ausführen, wobei ein bestimmter Gegenstand, auf den durch eine Stoffbezeichnung referiert wird, im Fokus steht, $d$. h. auch im bildlichen Zentrum des Videos, während die Personen selbst nur teilweise sichtbar sind. Die Videos sind dabei bewusst so kurz wie möglich gehalten, um den Gegenstand und nicht die Handlung in den Vordergrund zu rücken (s. Abb. 3). Die Aufgabenstellung lautet:

- EM3: Beschreiben Sie möglichst genau was die Frau (bzw. der Mann) im folgenden Video tut. [Video wird gezeigt]

Der Vorgang des Geldabhebens (EM3 SPT-S1 / SPT-D2) soll dabei einen eher generischen Kontext für Geld evozieren, da in dieser Konstellation nicht von einer bestimmten Summe oder Portion ausgegangen wird. Im Gegensatz dazu wird beim Vorgang des Lederschneidens (häufig wurde hier auch das Nomen Stoff von den GWP verwendet) ${ }^{28}$ ein konkretes Stück Leder geschnitten (EM3 SPT-S3 / SPT-D3). 

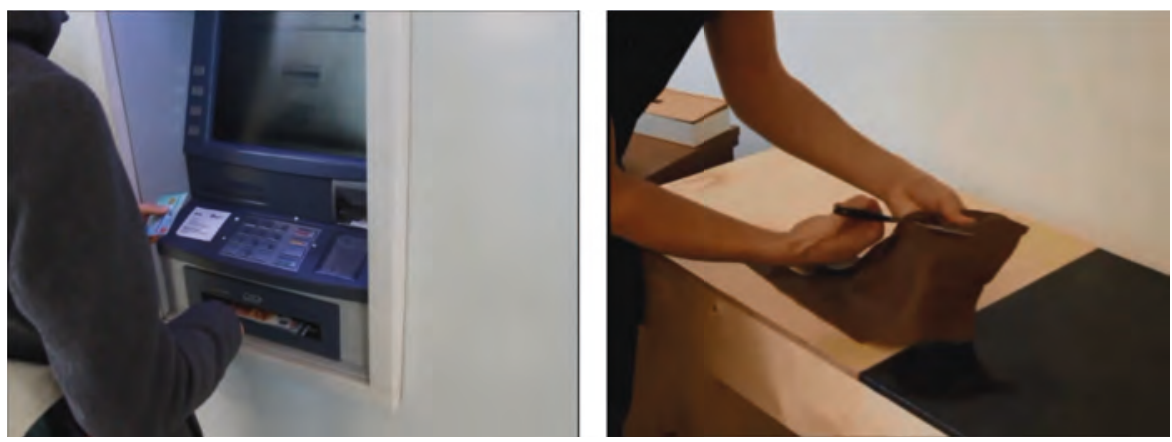

Abb. 3: Beispiel EM3 SPT-S1, EM3 SPT-D3

EM3 liefert GWP dabei die Möglichkeit, mit mehr als einem Satz zu antworten. Die meisten GWP nutzen diese Möglichkeit und beschreiben genauer, was in dem Video passiert, also geben z. B. an, dass der Pin-Code eingetippt oder auf welche Art das Leder geschnitten wird. Außerdem kann auf konkrete Gegenstände, mit denen etwas passiert bzw. die bereits in einem vorherigen Satz von den GWP genannt wurden und somit im Text nicht mehr neu sind, mit definitem Artikel referiert werden.

\section{Ergebnisse}

Die in den vorangehend beschriebenen SPT erhobenen Daten können aus vielen Perspektiven ausgewertet werden, wobei sich die Ausführungen hier auf die Primär-Variable des "unbestimmten Artikels vor Massennomen“ beschränken; außerdem werden im vorliegenden Artikel nur Frequenzanalysen geboten. ${ }^{29}$ Das heißt, dass aus den insgesamt 579 als relevant gewerteten Belegen ${ }^{30} 543$ ausgewertet werden, und zwar jene, die 0- oder EIN- vor einem Massennomen belegen. Die hohe Anzahl dieser Belege spricht dafür, dass die angewandten Aufgaben geeignet sind, um genau diese zu evozieren, d. h. auch der Kontext die Indefinitheit bzw. Neuheit der Referenten

29 Die Frequenzanalysen bieten natürlich nur einen ersten Blick auf die Ergebnisse und liefern vor allem methodische Anhaltspunkte. Nichtsdestoweniger können Aussagen über die Verteilung der Varianten getroffen werden. Weitere statistische Analysen wie Signifikanztests, Clusteranalysen etc. werden in weiteren Untersuchungen folgen, selbstverständlich auch in der Dissertation.

30 Als "relevant" wurden all jene Antworten gewertet, die ein Massennomen enthalten. Es wurden zudem weitere Belege aufgenommen, die für das Dissertationsprojekt relevant sind, $d$. $h$. dass sie andere syntaktische Variablen enthalten, die untersucht werden. Diese sollen hier aber nicht näher betrachtet werden. 
(s. o.) begünstigt. Abb. 4 zeigt die Verteilung aller Varianten (nach Häufigkeit geordnet, Erklärung s. u.), die insgesamt häufiger als 20-mal belegt sind. ${ }^{31}$

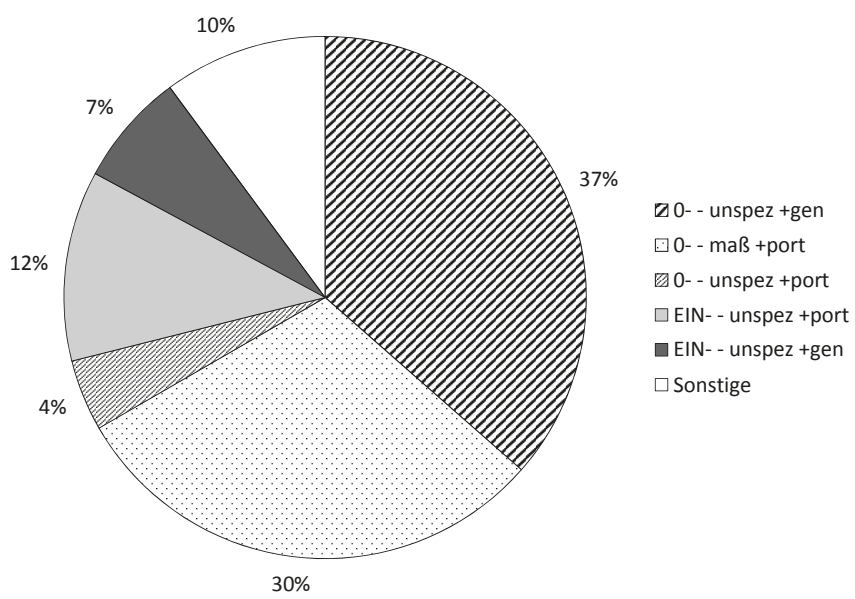

Abb. 4: Relative Häufigkeiten (in \%, $n=543$ ) der häufigsten Varianten in allen Aufgaben

In Abb. 4 sieht man, dass in den SPT insgesamt mehr als ein Drittel der Belege mit 0- und ohne Maßangabe auftritt, in unter einem Viertel der Belege wird hingegen EIN- verwendet, d. h., es kann eine starke Präferenz der standardsprachlich näheren Form festgestellt werden. Besonders auffällig ist, dass sich die Variante 0- mit Maßangabe mit rund einem Drittel der Belege in den Ergebnissen niederschlägt: Diese Variante ist jedoch v. a. im Kontext der Portionenlesart zu erwarten, also jenem Kontext, in dem gerade auch EIN- vor Massennomen auftreten soll. 0 - ohne Maßangabe ist in weniger als einem Viertel der Antworten belegt. Die Verteilung wird im Folgenden anhand der in Tab. 5 aufgelisteten und mit Beispielen aus den Antworten versehenen Varianten näher betrachtet. Die Variantenkürzel zeigen an, dass EIN- / 0- in der NP des Massennomens realisiert wurde, woraufhin angegeben wird, ob das Massennomen durch eine Form der Attribuierung (,attribuiert"), durch eine Maß-/Behälterangabe („maß“) oder gar nicht spezifiziert („unspez“) ist.

31 +gen und +port wird nur in dieser Abbildung verwendet und steht dafür, in welcher Lesart die Variante auftaucht. 


\begin{tabular}{|l|c|l|}
\hline Symbol & Kürzel & \\
\hline$\square$ & EIN- - maß & Die Laura hat ein Glasl mit einer länger frischen Vollmilch [...]. \\
\hline$\square$ & EIN- - attribuiert & Der Mann da tut ein braunes Leder zuschneiden. \\
\hline$\square$ & EIN- - unspez & Der Alex mag am liebsten ein Wasser. \\
\hline$\square$ & $0-$ - maß & Der Jakob hat ein Glas Wasser bestellt. \\
\hline$\square$ & 0- - attribuiert & Die Laura mag am allerliebsten frischen Kaffee. \\
\hline 0- - unspez & Jakob mag am liebsten Milch. \\
\hline
\end{tabular}

Tab. 5: Untersuchte Varianten mit Beispielen

Zunächst stellt sich die Frage, ob bezogen auf die Lesart Differenzen in der Variantenwahl festzustellen sind, $d$. h. eine Auswertung bezogen auf die angenommenen Steuerungsfaktoren (s. o.).

\begin{tabular}{|c|c|c|}
\hline \\
\hline $90 \%$ & & \\
\hline $80 \%$ & & \\
\hline $70 \%$ & & \\
\hline $60 \%$ & & \\
\hline $50 \%$ & & \\
\hline $40 \%$ & & \\
\hline $30 \%$ & & \\
\hline $20 \%$ & & \\
\hline $10 \%$ & & \\
\hline $0 \%$ & & \\
\hline & tgen & + port \\
\hline$\square$ EIN- - maß & & 1 \\
\hline$\square$ EIN- - attribuiert & 5 & 12 \\
\hline$\square$ EIN- - unspez & 38 & 64 \\
\hline$\square 0--m a ß$ & 12 & 164 \\
\hline $\mathbb{Q} 0$ - - attribuiert & 23 & 2 \\
\hline \$0- - unspez & 198 & 24 \\
\hline
\end{tabular}

Abb. 5: Relative Häufigkeiten der Variantenwahl (in \% und absoluten Zahlen, $n=543$ ) nach Lesart

In Abb. 5 zeigt sich ein sehr klares Bild der Präferenz von 0- ohne Maßangabe in der generischen Lesart $(40,7 \%)$ im Gegensatz zur Portionenlesart $(4,8 \%)$. Dies zeigt deutlich, dass ein Großteil der GWP in der generischen Lesart die standardnahe Variante bevorzugt. Dennoch wird in 7,9\% der Belege in der generischen Lesart EIN- und somit die standardfernere 
Variante realisiert; die Verwendung von EIN- steigt außerdem bei der Portionenlesart auf $14,0 \%$. Gerade bei der Portionenlesart wäre ein höherer Einsatz von EIN- zu erwarten, die meisten Belege wurden hier allerdings mit 0 - und einer Maßangabe realisiert (32,4 \%). Dies kann ein Indiz dafür sein, dass im (artifiziellen) SPT-Setting der Referent besonders genau beschrieben wird, kann aber auch darauf hinweisen, dass eine Verwendung mit EIN- auch in dieser Lesart vermieden wird. Um die Verteilung von 0- und EIN- genauer zu betrachten, werden Belege mit Maßangabe im Folgenden vernachlässigt, wodurch die Zahl der betrachteten Belege auf 366 sinkt. Nachdem geklärt wurde, wie sich der Steuerungsfaktor Lesart auf die Variantenwahl auswirkt, soll nun der situative Gebrauch der Varianten (intersituativer Vergleich) betrachtet werden.

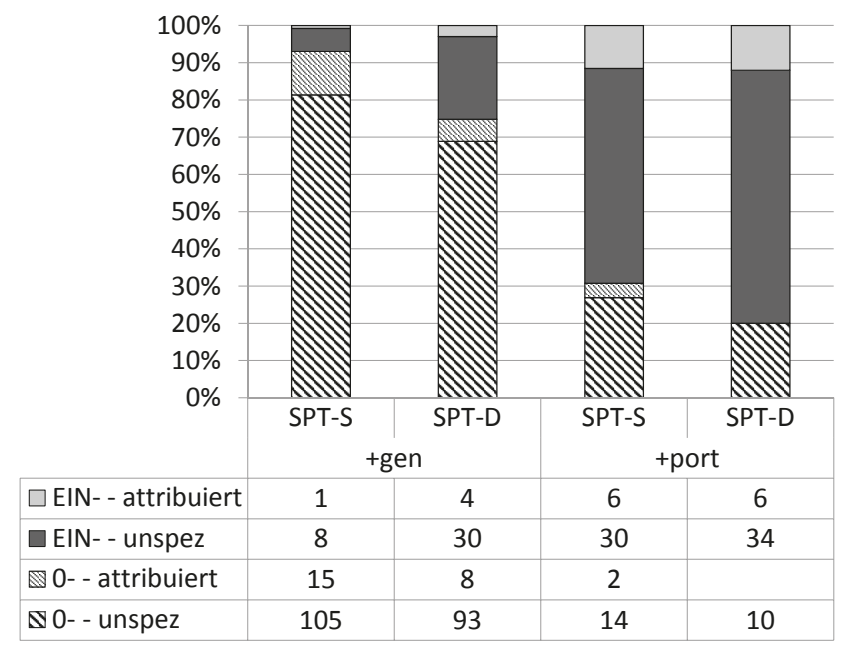

Abb. 6: Relative Häufigkeiten der Variantenwahl (in \% und absoluten Zahlen, $n=366$ ) intersituativ

Abb. 6 zeigt nach der Vernachlässigung der Belege mit Maßangabe deutlich, dass die Verwendung von EIN- nicht nur von der Lesart abhängig ist. Die Verwendung von EIN- steigt in der generischen Lesart von 7,0 \% im SPT-S auf $25,2 \%$ im SPT-D an, also um rund 18,0 \%; in der generischen Lesart von 69,2 \% auf 80,0 \% um rund $11,0 \%$. Das heißt, dass eine tendenzielle Steigerung der Gebrauchsfrequenz der standardferneren Varianten im SPT-D bei den GWP feststellbar ist - diese konnte bereits im durchgeführten Online-Fragebogen (s. Breuer 2016) belegt werden, dort allerdings 
in einem höheren Ausmaß, was insbesondere durch die Vorgabe von Varianten in den Bewertungsaufgaben erklärbar ist. Dabei muss berücksichtigt werden, dass gerade in der Portionenlesart die Maßangabe die dominante Variante ist, obwohI EIN- in diesem Kontext keine standardferne Variante darstellt. Bemerkenswert sind in diesem Zusammenhang die konsequente Verwendung von 0- einiger GWP sogar in diesem Kontext und der dennoch vorhandene (leichte) Anstieg der Gebrauchsfrequenz von EIN- im Dialektdurchlauf. Dies gibt nur einen schwachen Hinweis darauf, dass der SPT-D standardfernere Varietäten/Sprechlagen evoziert haben könnte, in denen EIN- die genauere Beschreibung durch die Maßangabe ersetzt. ${ }^{32}$ Zunächst lässt sich festhalten, dass bei dieser Variable Variation feststellbar ist - insbesondere ist dies bei der generischen Lesart hervorzuheben, da hier der EIN-Gebrauch bei standardnahen Varietäten/Sprechlagen sehr restringiert ist (s. o.). Die Frage ist nun, ob sich Verwendungspräferenzen der verschiedenen GWP-Gruppen feststellen lassen und hier deutlichere Tendenzen beobachtet werden können.

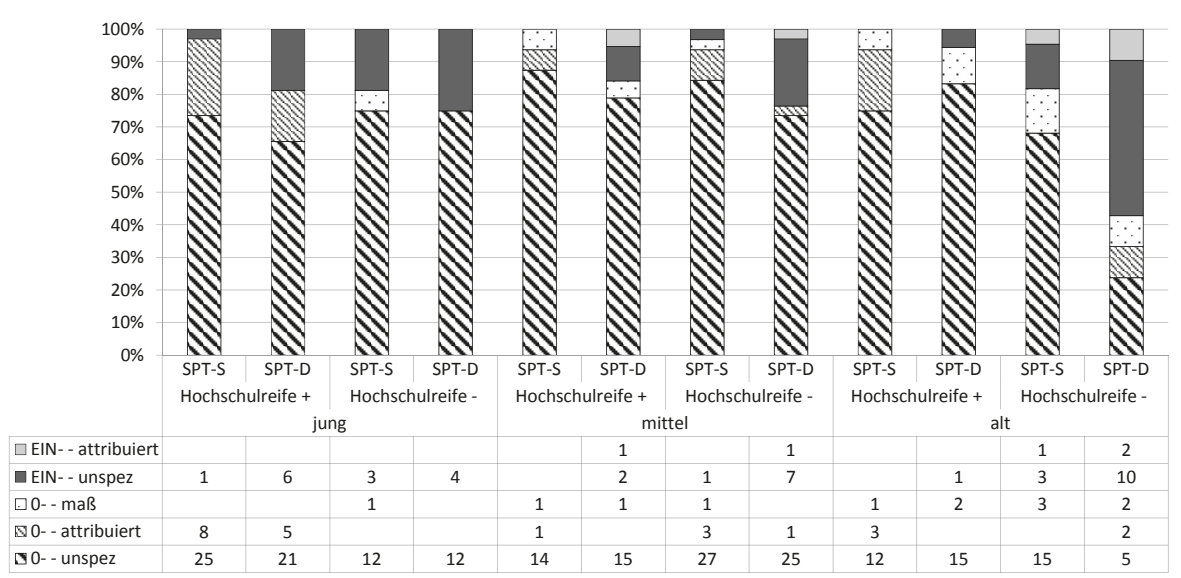

Abb. 7: Relative Häufigkeiten der Variantenwahl bei generischer Lesart (in \% und absoluten Zahlen, $n=276$ ) interpersonell

32 Als spannendes Sekundärphänomen hat sich die Verwendung von Diminutiv bei der Maßangabe (Glasl, Packl) herausgestellt. Die Verwendung der Diminutivform steigt im SPT-D deutlich an. 
In Abb. 7 wird der interpersonelle Vergleich bezogen auf die GWP-Gruppen (nach Alter und formalem Bildungsabschluss, s. o.) mit Bezug auf die generische Lesart (inklusive der Varianten mit Maßangabe) präsentiert. Viel deutlicher als in Abb. 6 können hier Tendenzen (trotz geringer Belegzahl je Personengruppe) zu Variantenfrequenzen gezeigt werden. In allen Gruppen zeigen sich zwei Tendenzen: Erstens steigt im SPT-D jeweils die Frequenz von EIN-, d. h. der standardferneren Variante. Außerdem verwenden GWP ohne Hochschulreife die Variante tendenziell häufiger als jene mit Hochschulreife. Sehr deutlich ist der situationsspezifische Gebrauch der Varianten bei der ältesten GWP-Gruppe ohne Hochschulreife zu erkennen. Während im SPT-S 0- mit 68,2 \% eindeutig die präferierte Variante ist, ist es im SPT-D EIN- mit $57,1 \%$. Daraus ergibt sich eine Differenz der Verwendung dieser Variante in den beiden Durchläufen von 38,9\%. Damit erweist sich diese Gruppe als die - situationsbezogen - dynamischste, also als die Gruppe mit der größten Variation. Interessanterweise erweist sich die Gruppe der ältesten GWP mit Hochschulreife komplementär dazu als jene mit der geringsten Dynamik, was auf eine mehr oder minder bewusste Vermeidung der standardferneren Variante hinweisen könnte.

\section{Resümee und Ausblick}

In gebotener Kürze lassen sich abschließend die Ergebnisse folgendermaßen zusammenfassen. Die für die Erhebung der syntaktischen Variable „unbestimmter Artikel vor Massennomen" entwickelten SPT sind geeignet, um das Phänomen zu untersuchen: Es werden eine Vielzahl an Belegen evoziert. Jene belegen einerseits eine starke Variation bedingt durch den innersprachlichen Steuerungsfaktor der Lesart, wobei 0- die dominante Variante in der generischen Lesart ist; dagegen sticht in der Portionenlesart die Variante mit Maßangabe hervor, aber auch die Gebrauchsfrequenz von EIN- steigt deutlich an. Auch die Situation bzw. der varietätenspezifische Stimulus bedingen Variation in der Variantenwahl, wobei 0 - häufiger in den SPT-S und EIN- vermehrt in den SPT-D auftritt. Anhand der interpersonellen Frequenzanalyse konnte gezeigt werden, dass insbesondere die GWP Gruppe alt + Hochschulabschluss intersituativ bedingt Varianten wählt: mit einer starken Präferenz von 0- bei den SPT-S und mit der Bevorzugung von EIN- bei den SPT-D.

Um alle im Projekt angelegten Variablen (soziodemographische, sprachlich kontextuelle, situative) zu überprüfen, müssen weitere Variablen herangezogen und untersucht werden, was im Zuge des Dissertationsprojekts vorgenommen wird. Dabei zeichnet sich bereits ab, dass die GWP-Gruppen je nach Variable eine unterschiedliche Dynamik aufweisen, was für die insgesamt hohe Dynamik des urbanen Varietätenkomplexes "Wienerisch“ 
spricht. Der Einbezug sekundärer Variablen verspricht eine weitere Verfeinerung der Ergebnisse und kann deren Validität sichern. Ebenso müssen Daten aus anderen Quellen herangezogen werden; vor allem qualitativ können sie durch den Einbezug der Interviews und Freundesgespräche abgesichert werden. Natürlich wäre auch ein Einbezug weiterer sprachlicher Systemebenen äußerst wünschenswert, kann aber forschungspragmatisch im Zuge des genannten Dissertationsprojekts nicht geleistet werden. Eine wiederverwendbare Aufbereitung der Daten und die Bereitstellung selbiger in Form einer (evtl. Online-)Datenbank sowie die (Langzeit-)Archivierung am Phonogrammarchiv gewährleisten, dass vielfältige weitere Untersuchungen des Materials möglich sind. Auch interdisziplinär werden damit wichtige Anknüpfungspunkte geschaffen.

\section{Literaturverzeichnis}

Breuer, Ludwig Maximilian \& Manfred M. Glauninger. 2012. „Editorial“. Tribüne 1: 2-3.

Breuer, Ludwig Maximilian. 2012. „Syntax in der Stadt, Sprachdynamisches ,Biotop“ Wien“. Tribüne 1: 8-12.

Breuer, Ludwig Maximilian. 2015. „Ganz Wien ist ein g'mischter Satz: Erforschung der syntaktischen Variation in Wien. Fallbeispiel ,unbestimmter Artikel vor Massennomen“". In: Lenz, Alexandra N., Timo Ahlers \& Manfred M. Glauninger (Hg.). Dimensionen des Deutschen in Österreich: Variation und Varietäten im sozialen Kontext. (Schriften zur deutschen Sprache in Österreich, 42). Frankfurt am Main: Peter Lang, 189-215.

Breuer, Ludwig Maximilian. 2016. „Methoden städtischer Regionalsprachenforschung: Wiener Variation des Relativsatzanschlusses im Online-Fragebogen“. In: Lenz, Alexandra N. \& Franz Patocka (Hg.). Syntaktische Variation - areallinguistische Perspektiven. (Wiener Arbeiten zur Linguistik, 2). Göttingen: V\&R unipress, 217-245.

Breuer, Ludwig Maximilian. i.V. „Wien, das was anders ist?: Relativsatz-Anschluss in einem spontansprachlichen ,Wiener' Korpus“. In: Lenz, Alexandra N. et al. (Hg.). Bayerisch-österreichische Varietäten zu Beginn des 21. Jahrhunderts - Dynamik, Struktur, Funktion. (ZDL-Beiheft). Stuttgart: Steiner.

Chierchia, Gennaro. 1998. „Plurality of mass nouns and the notion of 'semantic parameter'”. In: Rothstein, Susan (Hg.). Events and Grammar. Dordrecht: Kluwer, 53-103.

Dingeldein, Heinrich J. 2002. „Methoden der Stadtsprachenforschung“. In: Gerner, Zsuzsanna, Manfred M. Glauninger \& Katharina Wild (Hg.). Gesprochene und geschriebene deutsche Stadtsprachen in Südosteuropa und ihr Einfluss auf die regionalen deutschen Dialekte: Internationale Tagung in Pécs, 30.3.2.4.2000. (Schriften zur diachronen Sprachwissenschaft, 11). Wien: Ed. Praesens, 21-37.

Fleischer, Jürg, Simon Kasper \& Alexandra N. Lenz. 2012. „Die Erhebung syntaktischer Phänomene durch die indirekte Methode: Ergebnisse und Erfahrungen aus dem Forschungsprojekt ,Syntax hessischer Dialekte' (SyHD)". Zeitschrift für Dialektologie und Linguistik 79: 1-42.

Gallmann, Peter. 1990. Kategoriell komplexe Wortformen: Das Zusammenwirken von Morphologie und Syntax bei der Flexion von Nomen und Adjektiv. (zugl.: Dissertation, Universität Zürich, 1989/90). Tübingen: Niemeyer.

Glaser, Elvira. 1996. „Morphologie und Funktion des unbestimmten Artikels im Bairischen“. In: Eroms, Hans-Werner \& Hermann Scheuringer (Hg.). Sprache an Donau, Inn und Enns: Vorträge des Symposiums zu Sprache und Kultur des altbairischen Raumes, zugleich Zweite Arbeitstagung zu Sprache und Dialekt in 
Oberösterreich, vom 12. bis 14. Mai 1995 auf Burg Neuburg am Inn. (Schriften zur Literatur und Sprache in Oberösterreich, 5). Linz: Adalbert-Stifter-Inst. des Landes Oberösterreich, 149-169.

Glauninger, Manfred M. 2008. „Synthetische und analytische ,Konjunktiv 2'-Formen im Wiener Nonstandard-Deutsch“. In: Patocka, Franz \& Guido Seiler (Hg.). Dialektale Morphologie, dialektale Syntax: Beiträge zum 2. Kongress der Internationalen Gesellschaft für Dialektologie des Deutschen. Wien, 20.-23. September 2006. Wien: Praesens, 233-247.

Glauninger, Manfred M. 2011. „Zum honorativen Konjunktiv 2 als Modalisierungsoption. Aspekte einer funktionalen Typologie des Wiener Deutsch“. In: Christen, Helen, Franz Patocka \& Evelyn Ziegler (Hg.). Struktur, Gebrauch und Wahrnehmung von Dialekt: Beiträge zum 3. Kongress der Internationalen Gesellschaft für Dialektologie des Deutschen (IGDD), Zürich, 7.-9. September 2009. Wien: Praesens, 47-57.

Glauninger, Manfred M. 2012. „Zur Metasoziosemiose des >Wienerischen<: Aspekte einer funktionalen Sprachvariationstheorie“. LiLi 42: 110-118.

Grammis. 2005. „Zur Bedeutung minimaler Nominalphrasen“. In: grammis 2.0: Systematische Grammatik. Zuletzt geändert am 2.10.2005. Online: <http://hypermedia.ids-mannheim.de/call/public/sysgram. ansicht?v_typ=d\&v_id=1608> (22.7.2016).

Kallenborn, Tim. 2011. „Ein Ansatz zur Erhebung regionalsprachlicher Syntax“. In: Christen, Helen, Franz Patocka \& Evelyn Ziegler (Hg.). Struktur, Gebrauch und Wahrnehmung von Dialekt: Beiträge zum 3. Kongress der Internationalen Gesellschaft für Dialektologie des Deutschen (IGDD), Zürich, 7.-9. September 2009. Wien: Praesens, 80-98.

Kallenborn, Tim. 2016. „Regionalsprachliche Syntax: Horizontal-vertikale Variation im Moselfränkischen“. Dissertation, Universität Wien.

Kolde, Gottfried. 1989. Der Artikel in deutschen Sachverhaltsnominalen. Tübingen: Niemeyer.

Kortmann, Bernd. 2010. „Areal Variation in Syntax“. In: Auer, Peter \& Jürgen Erich Schmidt (Hg.). Language and Space. Vol. I: Theories and Methods. (Handbücher zur Sprach- und Kommunikationswissenschaft, 30.1). Berlin: de Gruyter, 837-864.

Krifka, Manfred. 1991. „Massennomina“. In: Stechow, Arnim von \& Dieter Wunderlich (Hg.). Handbook of Semantics. Berlin: de Gruyter, 399-417.

Lenz, Alexandra N. 2003. Struktur und Dynamik des Substandards: Eine Studie zum Westmitteldeutschen (Wittlich/Eifel). Stuttgart: Steiner.

Lenz, Alexandra N. 2008. „Wenn einer etwas gegeben bekommt: Ergebnisse eines Sprachproduktionsexperiments zum Rezipientenpassiv“. In: Patocka, Franz \& Guido Seiler (Hg.). Dialektale Morphologie, dialektale Syntax: Beiträge zum 2. Kongress der Internationalen Gesellschaft für Dialektologie des Deutschen; Wien, 20.-23. September 2006. Wien: Praesens, 157-180.

Moosmüller, Sylvia. 1987. Soziophonologische Variation im gegenwärtigen Wiener Deutsch: Eine empirische Untersuchung. (zugl.: Dissertation, Universität Wien, 1984 u.d.T.: Soziale und psychosoziale Sprachvariation). Stuttgart: Steiner.

Moosmüller, Sylvia. 1991. Hochsprache und Dialekt in Österreich: Soziophonologische Untersuchungen zu ihrer Abgrenzung in Wien, Graz, Salzburg und Innsbruck. Wien: Böhlau.

Niebaum, Hermann \& Jürgen Macha. 2006. Einführung in die Dialektologie des Deutschen. 2., neubearb. Auflage. (Germanistische Arbeitshefte, 37). Tübingen: Niemeyer.

Patocka, Franz. 1989. „Dialektsyntax und Syntaxgeographie: Möglichkeiten und Grenzen“. In: Putschke, Wolfgang, Werner Veith \& Peter Wiesinger (Hg.). Dialektgeographie und Dialektologie: Günter Bellmann zum 60. Geburtstag von seinen Schülern und Freunden. Marburg: Elwert, 47-56.

Patocka, Franz. 1997. Satzgliedstellung in den bairischen Dialekten Österreichs. (Schriften zur deutschen Sprache in Österreich, 20). Frankfurt am Main etc.: Lang.

Peter, Klaus. 2015. „Sprachliche Normvorstellungen in Österreich, Deutschland und der Schweiz“. In: 
Lenz, Alexandra N., Timo Ahlers \& Manfred M. Glauninger (Hg.). Dimensionen des Deutschen in Österreich: Variation und Varietäten im sozialen Kontext. (Schriften zur deutschen Sprache in Österreich, 42). Frankfurt am Main: Lang, 123-147.

Reinecke, Jost. 2014. „Grundlagen der standardisierten Befragung“. In: Baur, Nina \& Jörg Blasius (Hg.). Handbuch Methoden der empirischen Sozialforschung. Wiesbaden: Springer VS, 601-617.

Schmidt, Jürgen Erich \& Joachim Herrgen. 2011. Sprachdynamik: Eine Einführung in die moderne Regionalsprachenforschung. (Grundlagen der Germanistik, 49). Berlin: Erich Schmidt.

Strobel, Thomas. 2016. „Indefinitartikel bei Kontinuativa“. In: SyHD-atlas. Online: <http://syhd.info/ apps/atlas/\#indefinitartikel-bei-kontinuativa> (22.7.2016).

Tatzreiter, Herbert. 2002. „Die Wiener Stadtsprache - innovativ und beharrsam“. In: Gerner, Zsuzsanna, Manfred M. Glauninger \& Katharina Wild (Hg.). Gesprochene und geschriebene deutsche Stadtsprachen in Südosteuropa und ihr Einfluss auf die regionalen deutschen Dialekte: Internationale Tagung in Pécs, 30.3.-2.4.2000. (Schriften zur diachronen Sprachwissenschaft, 11). Wien: Ed. Praesens, 127-138.

Weiß, Helmut. 1998. Syntax des Bairischen: Studien zur Grammatik einer natürlichen Sprache. Tübingen: Niemeyer.

Zifonun, Gisela. 2007. Grammatik des Deutschen im europäischen Vergleich: Das Pronomen. Teil IV: Indefinita im weiteren Sinne. (Amades, 4/06). Mannheim: Institut für Deutsche Sprache.

\section{Abkürzungsverzeichnis}

0- $\quad$ 0-Artikel bzw. Fehlen eines Artikels

D- Bestimmter Artikel (der, die, das)

EIN- Unbestimmter Artikel (ein, eine)

GWP Gewährsperson

maß Maß-/Mengenangabe

SPT Sprachproduktionstest

SPT-D Sprachproduktionstest - Dialektdurchlauf

SPT-S Sprachproduktionstest - Standarddurchlauf

unspez Unspezifiziertes Auftreten eines Massennomens

Ludwig Maximilian BREUER:

Institut für Germanistik, Universität Wien

http://www.Imbreuer.de

ludwig.maximilian.breuer@univie.ac.at

doi: 10.1553/jpa7s86-111 